\title{
ESTADO VEGETATIVO-ALFA PERSISTENTE
}

\author{
R. OLIVEIRA-SOUZA* - D. L. GUSMAO**- W. M. FIGUEIREDO *** \\ I. R. R. A. LÓPEZ****; - J. S. MELLO*:** - S. A. RIBEIRO*- H. ALVARENGA*
}

\begin{abstract}
RESUMO -- Após revisão sumária dos conceitos de «coma» e «coma-alfa», relatamos o caso de uma paciente com doença de Pick provável que, nos estágios terminais da doença, permaneceu várias semanas em estado vegetativo com EEG de vigilia normal e reativo à fotoestimulaçāo. Enfatizamos (a) algumas das implicações do aspecto dos olhos nesses pacintes, (b) a importância de distinguir a atividade alfa do ritmo alfa verdadeiro e (c) sugerimos que a categoria «coma-alfa» inclua apenas aqueles com afecçoes do tegmento da junção pontomesencefálica, de qualquer natureza, reservando a designaçâo «estado vegetativo-alfa» persistente para os que se encontrem em estado vegetativo e exibam EEG em virgilia idêntico ao normal.
\end{abstract}

Persistent alpha-vegetative state.

SUMMARY - After briefly reviewing the concepts of «coma» and «alpha-coma», we report the case of a patient with presumable Pick's disease who spent several weeks in a vegetative state with a normal and reactive EHG in the alpha range in the later stages of her illness. We (a) emphasize some implications of the appearence of the eyes in such patients, b) stress the importance of distinguishing alpha activity from true alpha rhythm and (c) suggest the category «alpha-coma» should include only those individuals bearing evidence of disorders of the junctional tegmentum of the pons and midbrain. In a complementary way, we propose that natients in a persistent vegetative state displaying normal EEG should be separately classified as persistent «alpha-vegetative state».

O coma tem sido modernamente conceituado como um estado de irresponsividade no qual o paciente jaz inerte de olhos fechados 87 . A condiçăo se acompanha de abolição do tônus postural e do controle esfincteriano, deixando o paciente desprovido de movimentos ativos e restringindo seu repertório motor a reflexos segmentares e multisegmentares: respiração, tosse, bocejos. Os casos mais 'puros', nos quais não se encontram sinais de lesóes localizadas no cérebro ou no tronco cerebral, são produzidos pelas intoxicações exógenas, devidas a álcool ou barbitúricos, por exemplo. $\mathrm{Na}$ prática, todavia, freqüentemente sinais como hemiplegia ou desvios da mirada coexistem no mesmo paciente, denunciando não só a presença de lesão circunscrita no encéfalo mas, também, o 'locus' presumivel de origem do próprio estado de coma. Observações clinicas e experimentais têm demonstrado que os indices semiológicos mais sensíveis para a aferiçāo da presença de vigília ('wakefulness') - e os primeiros a desaparecerem no coma ou na sonolência fisiológica ou patológica - são a abertura dos olhos, efetuada, sobretudo, pela contração dos músculos elevadores das pálpebras superiores, e o tônus dos músculos axiais, em especial dos extensores da cabeça (Oliveira-Souza R., observações não publicadas). Esses músculos recebem inervação, respectivamente, dos nervos oculomotores e do plexo cervical. Os dois músculos eleva-

* Serviço de Neurologia, Hospital Universitário Gaffrée e Guinle, UNI-RIO, Rio de Janeiro (HUGG); ** Ex-Residente de Neurologia. Santa Casa de Misericórdia, Rio de Janeir’o; *** Serviço de Clinica Médica III, HUGG; **** Hospital Dr Philippe Pinel, DINSAM-MS, Rio de Janeiro (HPP); ***** Serviço de Epilepsia e Eletrencefalografia, HPP. 
dores das pálpebras são inervados pela divisão única e mediana dos núcleos do terceiro par craniano — denominada 'subnúcleo centrocaudal' —, localizada dorsalmente ao terço caudal dos complexos nucleares oculomotores comuns 56 . A caracterização do estado de coma como simples abolição da vigilia pôde ser formulada em bases fisiológicas mais consistentes a partir da identificação do sistema reticular ativador ascendente (SRA), situado no tegmento do tronco cerebral, por Moruzzi e Magoun 75 e por Lindsley, Bowden e Magoun 63, em 1949. Uma vez que o subnúcleo centrocaudal é contíguo a formaçāo reticular tegmental, a ocorrência de atividade funcional nesta provavelmente se traduz, de imediato, pela contração dos elevadores das pálpebras. Portanto, hoje em dia, à equação semjológica: "coma $=$ irresponsividade + olhos fechados', denotativa de ausência de vigília, pode-se ajuntar outra, de natureza fisiopatológica: 'coma $=$ abolição patológica de função SRA'. Fora dos limites da validade clinica dessas equações encontram-se a síndrome de encarceramento mesencefálica 73 e alguns casos raros de polineuropatia pós-infecciosa 10 , curarização, crise miastênica e paralisias calêmicas periódicas, cujo reconhecimento dependerá de alto grau de desconfiança diagnóstica. Antes de 1949, estudos esporadicos em seres humanos já haviam conduzido à generalização de que um traçado eletrencefalográfico (EEGráfico) lento, irregular, continuo e de alta voltagem era característico dos estados de coma, análogo ao então observado no sono natural 107. Com a formulação do conceito de SKA, empreenderam-se novas investigaçōes voltadas para os padrões EEGráficos nos estados de coma por lesões estruturais. A previsão de que pacientes em coma exıbırıam traçados sıncronızados tol, efetivamente, confirmada, prıncıpalmente para determinado grupo de individuos levados ao coma por destruição ou compressão do tegmento mesencefálico 27,80 ou da região subtálamo-talâmica (transição mesodiencefálica $)^{5,6,19,64,70,91,96,102}$, regiōes estas compreendidas dentro dos limites do território das artérias basilares comunicantes ou mesencefálicas 94,96. Muitos outros pacientes em coma manifestavam, ao contrário, padrões EEGráficos diferentes do anterior, descritos por Jasper e van Buren 50, Loeb 64, Loeb et al.66 e Silverman 57 , contrariando as expectativas iniciais. De modo geral, esses estudos acabaram refutando o dogma da 'congruência eletroclínica', complementando-o com a idéia de 'dissociaçāo eletroclínica', isto é, da ausência de qualquer relaçāo necessária ou constante entre o grau normal ou patológico de redução da vigília e a quantidade ou o tipo de modificações EECiráficas concomitantes. Esse conceito tirmou-se em definitivo com o reconhecimento do estágio KEM do sono tisiológico - cuja autenticidade lhe é outorgada precisamente pela notável dissociaçăo eletroclínica registrada 53 - e dada a possibilidade de se criarem estados artificiais de dissociação análogos em animais de experimentação, quer por meios fisiológicos 4 ou farmacológicos 93 . Na verdade, a origem da noção de dissociação eletroclínica pode ser rastreada ao caso relatado por Loeb e Poggio em 195365. Tratava-se de uma paciente em coma devido a hemorragia no tegmento da protuberância que, ao eletroencefalograma (EEG), apresentava ritmo bastante regular de \$-9 cps, alguma atividade rápida bifrontal de baixa voltagem e recrutamento bilateral dos ritmos cerebrais pela estımulação fótica intermitente'. Estudo angiográfico demonstrou a patência das artérias cerebrais posteriores. Relatos semelhantes se seguiram 13,45,54,68,81.84,89,110 e, em 1975, Westmoreland et al.109 propuseram a designaçāo 'coma-alfa' à 'conjunção de coma clínico com um padrão eletrencefalográfico assemeIhando-se àquele da vigília normal'. Reconheceram dois grupos de pacientes assim definidos. $O$ primeiro consistia de indivíduos acometidos de afecções vasculares do tronco cerebral, na altura ou logo abaixo da junçāo pontomesencefálica (ou istmo do tronco cerebral), comprometendo estruturas nutridas por ramos protuberancias da artéria basilar 67. O segundo grupo, ao contrário, compunha-se de casos de encefalopatia pós-anóxica, na maior parte ocasionada por parada cardiorrespiratória, com envolvimento predominante das camadas infragranulares ( $\mathrm{V}$ e VI) do córtex cerebral e preservação relativa das camadas supragranulares (II e III). Além disso, encontravam-se igualmente pouco afetados núcleos da base, tálamo e tronco cerebral 8,14,38,105. Estudos EEGráficos repetidos têm revelado algumas diferenças notáveis entre os dois grupos. Os traçados daqueles em coma-alfa por lesão do tronco cerebral apresentam maior variabilidade espontânea e reatividade frente a estimulação aferente. Além disso, mostram-se mais uniformes em registros seriados. Nesses casos, observações poligráficas contínuas $7,28,44$ demonstram que a atividade alfa captada em ocasiōes diferentes pode dar lugar, segunoo padrao ue alternancia quase circadiana, a ritmos lentos e fusos-de-sono jdênticos aos encontrados na fase 2 do sono não-REM de adultos normais e, como tais, reativos a estimulação periférica, que prontamente os reverte para a faixa alfa ou beta. Em contraste, a atividade alfa obtida na encefalopatia pós-anóxica é transıtoria, pouco reatıva e variável, manifestando-se na primeira semana da parada cardiorrespiratória para ceder a vez, nos dias subseqüentes, às alterações EEGráficas que prenunciam a morte. O 'ritmo alfa', propriamente dito, das lesōes 
do istmo do tronco cerebral, tem se revelado idêntico ao encontrado em condiçóes normais 49 , ao passo que a atividade alfa gerada na encefalopatia pós-anóxica parece depender de mecanismos fisiopatológicos anômalos, essencialmente distintos de qualquer padrăo vigente em situação de normalidade 29,31,48. Estudos experimentais indicam que, nesse caso, a atividade alfa anômala resulta da projeção à distância para o córtex de ritmos instáveis gerados na amigdala temporal, transmitidos por meio de determinados núcleos talâmicos 38 , ao passo que, em se tratando do ritmo alfa genuíno 49 , o sistema tálamo-cortical é recrutado por projeçóes ascendentes a partir de regiões especificas do tegmento do tronco cerebral 4,12,63.

$\mathrm{Na}$ tabela I encontram-se citadas as principais etiologias conhecidas do coma-alfa e condiçōes aparentadas. O caso a seguir representa mais uma alternativa etiológica para este grupo de condições, ainda não devidamente enfatizada na literatura especializada.

1. Pós-anóxico (superada a fase aguda, mais propriamente caracterizado como 'estado vegetativo'):

- parada cárdio-respiratória $(2,8,14,35,37,48,60,61,83,103,105,109)$

- estrangulamento (15)

- quase-afogamento (4j)

- pós-descarga elétrica de alta voltagem $(34, .55)$

2. Afecçōes vasculares do tegmento da transiçāo pontomesencefálica (istmo) * $(5,12,13,35$, $41,54,65,68,81,83,84,89,91,103,109,110)$

3. Intoxicaçāo medicamentosa $(11,24,39,58,59,61,69,88)$ :

- barbitúricos

- benzodiazepinicos

- neurolépticos

- antidepressivos tricíclicos

- glutetimida (năo comercializada no Brasil)

4. Pós-traumático $(7,81,103,104)$

5. Estados hiperosmolares $(1,2)$

6. Enxaqueca da artéria basilar (25)

7. Morte encefálica ou troncular (33)

8. Demência do tipo lobo frontal $(7 \%, 78)$ (variante 'clâssica' da doença de Pick, 76 ):

- presente caso (como em 1, a designação 'estado vegetativo' parece mais apropriada)

Trabela 1 - Classificação etiológica do coma-alfa e dos estados afins. * Estes pacientes parecem representar o grupo 'genuino' de comas-alfa por exibirem, aliado a 'irresponsividade de olhos fechados', um 'ritmo alfa' com atributos idsnticos aos daqueles captados $\mathrm{em}$ pessous normais $(12,29,31,44,49,65)$. Situação identica pode ocorrer em pacientes pertencentes aos grupos 3,4 e 6 .

\section{OBSERVAÇAO}

Uma muther preta (MGO), com 60 anos de idade, foi recolnida em via pública por desconhecido e conduzida em estado de abandono e higiene precária ao Hospital Dr Philippe Pinel, onde permaneceu internada durante um ano e quatro meses. Desde a internação, em maio/1985, mostrou-se desorientada, incapaz de dizer quem era, o que lhe sucedera nos últimos tempos, onde se encontrava. Não apresentava aiteraçōes sistêmicas, conservando pressão arterial em $110 \times 70 \mathrm{mmHg}$, freqüência cardiaca regular ( 80 bpm), glicemia de $90 \mathrm{mg} \%$. hemograma, VHS e RX de tórax normais. Confusa, recusava-se cooperar com as equipes d: enfermaria, ainda que para cumprir solicitaçöes simples, preferindo recolher-se ao leito e cobrir a cabeça com o lençol, respondendo com monossilabos ou evasivas às perguntas a ela enderecadas. Freqüentemente evitava contato com as companheiras de grupo de terapia ocupacional para procurar argila, tijolos de barro e até lixo e terra, que levava à boca e deglutia. Em outubro, foi transferida para instituição geriátrica com diagnóstico de demência 
senil. Retornou dias depois por năo haver obtido «adaptação satisfatória»: agredia as companheiras de enfermaria, principalmente quando protestavam contra suas intromissões na mobilia ou nos objetos de uso pessoal. Várias vezes, surpreenderam-na tentando sair para a rua, o que se interpretava como «tentativas de fuga». Ainda que falasse muito potico, foi medicada com $6 \mathrm{mg}$ diários de haloperidol e biperideno, no início de novembro, por exibir atividade deambulatória quase incessante. Duas semanas depois, passou a não mais se alimentar de modo satisfatório, emagrecendo e manifestando sinais de impregnação neuroléptica. Suspensa a medicaçăo, recuperou o nível prévio de atividade em menos de uma semana. Desde então, a única medicaçāo que recebeu foram 10 mg de diazepam à noite. Nesta ocasiāo, $j a ́$ se encontrava em mutismo quase absoluto, incapaz de estabejecer qualquer tipo de comunicacão inteligível. Nova rotina laboratorial revelou estarem normais hemograma, $\mathrm{Rx}$ de tórax e níveis plasmáticos de glicose, uréia e creatinina. $\mathrm{O}$ olhar vago e fixo coincidia com a inexpressividade do semblante. Nåo obstante, movimentava-se praticamente durante todo o período de vigília, deambulando, cabisbaixa, a passos lentos, sentarido, deitando, pondo-se de pé outra vez e reiniciando a caminhada a esmo. Por vezes interrompia tal perambulação despropositada a intervalos irregulares para manipular com desinteresse objetos casualmente dispostos em seu trajeto, os quais eram eventualmente ingeridos, ou para permitir a eliminação de fezes e urina, atos sobre os quais nāo mais detinha o menor controle. No decorrer da primeira metade de 1986, o estado demencial prosseguiu se agravando. Em fins de agosto, a ativıdade deambulatória não mais existia e a paciente consumia a maior parte do tempo deitada no leito, o que tavoreceu o desenvolvimento de escaras de decúbito e perda ponderal adicional. Permanecia, no entanto, isenta de complicaçoes infecciosas ou metabólicas. Nesse estado, foi transferida para hospital geral, para acompanhamento clínico.

Em setembro/1986, deparamo-nos com a paciente pela primeira vez. Encontrava-se inıóvel e muda, deitada em atitude de descorticacāo bilateral, desvio sustentado da cabeça e dos olhos para a direita, sem movimentos ativos, respirando regularmente cerca de 16 vezes por minuto. Frequentemente fechava os olhos por longos períodos, aparentando dormir. Nesta condição, abria-os de pronto, tāo logo submetida a estimulaçāo cutânea não nociceptiva, de intensidade moderada. Uma vez desperta, dirigia o olhar para a face do examinador, dela situado a menos de dois metros, passando a acompanhá-lo de modo lento e contínuo, ou a qualquer outro estímulo suficientemente intenso ou inesperado que porventura invadisse seu campo visual. Esse seguimento era feito com a mirada horizontal, principalmente para a direita, a partir da linha média. Com dificuldade, perfazia movimentos para a esquerda. Nas miradas extremas, conjugavam-se à mirada movimentos vagarosos de rotação lateral da cabeça para o mesmo lado. Se o examinador se deslocasse na direção dos seus pés, ocasionalmente rebaixava o olhar e fletia a cabeça devagar, assim permanecendo por instantes. Jamais se registraram convergência ou elevação dos olhos. Todos os movimentos de acompanhamento eram iniuterruptos e conjugados, observando-se deslocamentos sacádicos esporádicos quando os olhos perdiam seu alvo móvel e regressavam, com a cabeça, à posição inicial de desvio para a direita. Nāo conseguimos atrair sua «atençāo visual» por meio de estímulos auditivos mas, às vezes, acompanhava com o olhar a manipulaçāo do seu braço direito. Permanecia de olhos abertos por periodos de tempo prolongados, durante os quais pestanejava cerca de 20 vezes por minuto, reagindo à ameaça visual com cclusão breve e completa das fendas palpebrais. No mais das vezes, porém, o pestanejar era incompleto, aparentemente produzido pela contração delicada da porção palpebral dos orbiculares dos olhos. Além disto, a estimulacão repetitiva com «filash» luminoso induzia sua habituacão. Com a aproximação do sono, as contraçōes do piscar-de-olhos tornavam-se progressivamente mais amplas e duradouras, somando-se umas às outras, até ocluirem definitivamente as fendas palpebrais. Os reflexos córneo-palpebrais estavam presentes e se acompanhavam de rotação associada dos olhos para cima (fenômeno de Bell), nāo se registrando contraçōes pterigóides concomitantes. As pupilas eram isocóricas ( $2 \mathrm{~mm}$ de diâmetro) e fotorreagentes, reflexos consensuais bilaterais. Os reflexos óculo-cefálicos estavam presentes e venciam com certa dificuldade o desvio tônico da mirada para a direita. quando a cabeça era bruscamente rodada para este lado. Os reflexos vestibulo-oculares (água fria) desviavam os olhos para o lado excitado. Quando se irrigava à direita, o desvio da mirada se acentuava ainda mais e, nesta ocasião, se os olhos se desviassem para o lado contrário por breves instantes, advinha nistagmo com fase rápida para a esquerda; quando se irrigava à esquerda, os olhos se desviavam nesse sentido, não ultrauassando a linha mediana das órbitas e apresertando nistagmo com fase rápida para a direita. A estimulaçāo simultânea induzia rebaixamento e desvio c.a mirada para a direita, com nistagmo horizontal prolongado. A face era simétrica, nāo expressando emoçōes ou reaçōes de dor ou desconforto, ainda que durante a manipulação da escara sacrococcigea que se aprofundara. Conservava a boca fortemente ocluída por 
contratura dos masséteres, que persistia na vigília e no sono aparente. Não exibia mastigaçãu espontânea automática, nem emitia som de qualquer espécie. Os reflexos axiais da face e o de retração da cabeça encontravam-se hiperativos, os glabelares inesgotáveis. Quando sentada, parecia mais «atenta» ao ambiente, apresentando movimentos oculares sacádicos com maior frequência, ainda que năo manifestasse a menor evidência de tônus postural ou reflexos de endireitamento no tronco ou nos membros. Nesta posiçāo, apareciam com maís nitidez reflexos de preensāo («grasping») e de persecução compulsória («groping») na mão esquerda. Os músculos esqueléticos apresentavam redução universal de massa e intensa contratura em descorticação, mais evidente no dimídio direito. A palpação, os músculos cervicais e axiais do tronco mostravam-se rígidos, deixando transparecer seus relevos sob a pele. Os dos membros, ao contrário, eram flácidos. Os reflexos musculares mostravam-se hiperativos $(6+/ 6+)$ e simetricos, os reflexos plantares forneciam resposta em flexāo à esquerda e em extensāo à direita. O EEG, realizado em eletrencefalógrafo Berger de 8 canais, evidenciou ritmo alfa de baixa voltagem, $11-13$ cps, regular, atenuável pela abertura passiva dos o!hos, predominando nas áreas occipitais, repetidamente interrompido pela atividade rápida dominante nas áreas anteriores (Fig. 1). A intensa contratura dos masséteres produzia artefatos

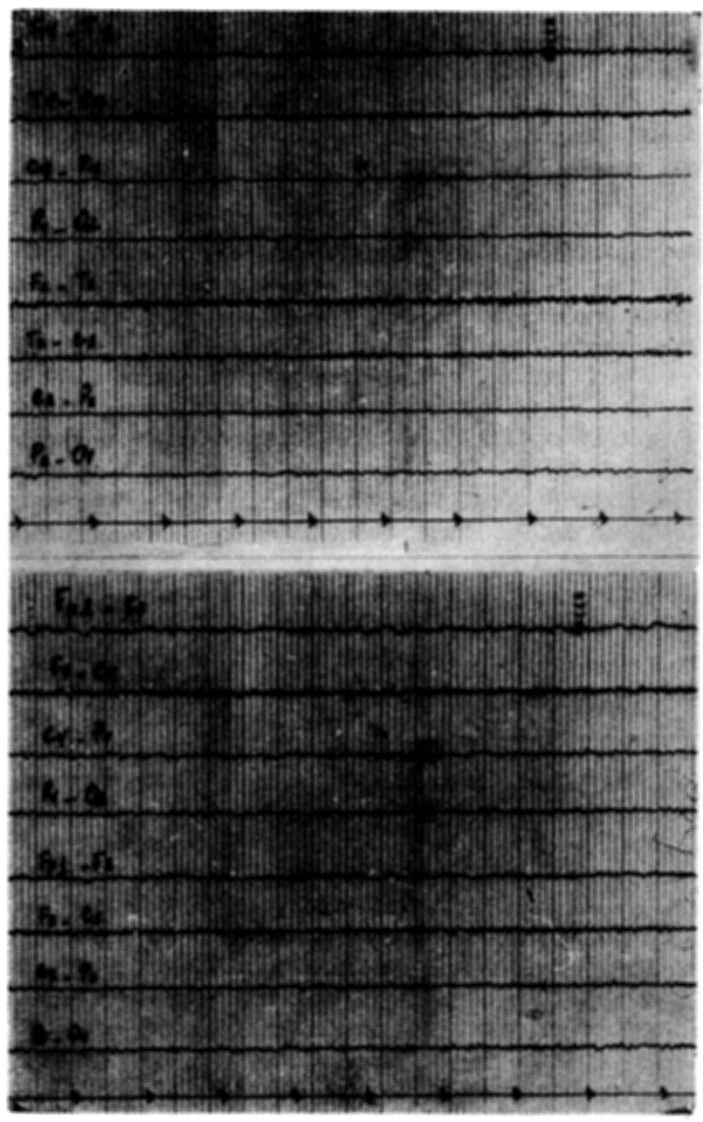

F'ig. 1 - Caso MGO: exame eletrencefalográfico.

que prejudicaram, em parte, a qualidade do traçado. A tomografia computadorizada do crânio (TC), realizada em tomógrafo EMI-Scanner CT 1010, matriz 320x320, cortes de 10mm de espessura, revelou parênquima encefálico isodenso e acentuado aumento dos ventrículos cerebrais, das fissuras sylvianas (principalmente do hemisfério esquerdo) e das cisternas suprasselar, mesencefálica e do vermis superior. Os sulcos corticais, em contrapartida, estavam apenas um pouco aumentados, difusamente (Fig. 2). A punção lombar deu saída a liquido cefalorraqueano (LCR) límpido, normotenso (130mm H2O) e incolor. O exame do 


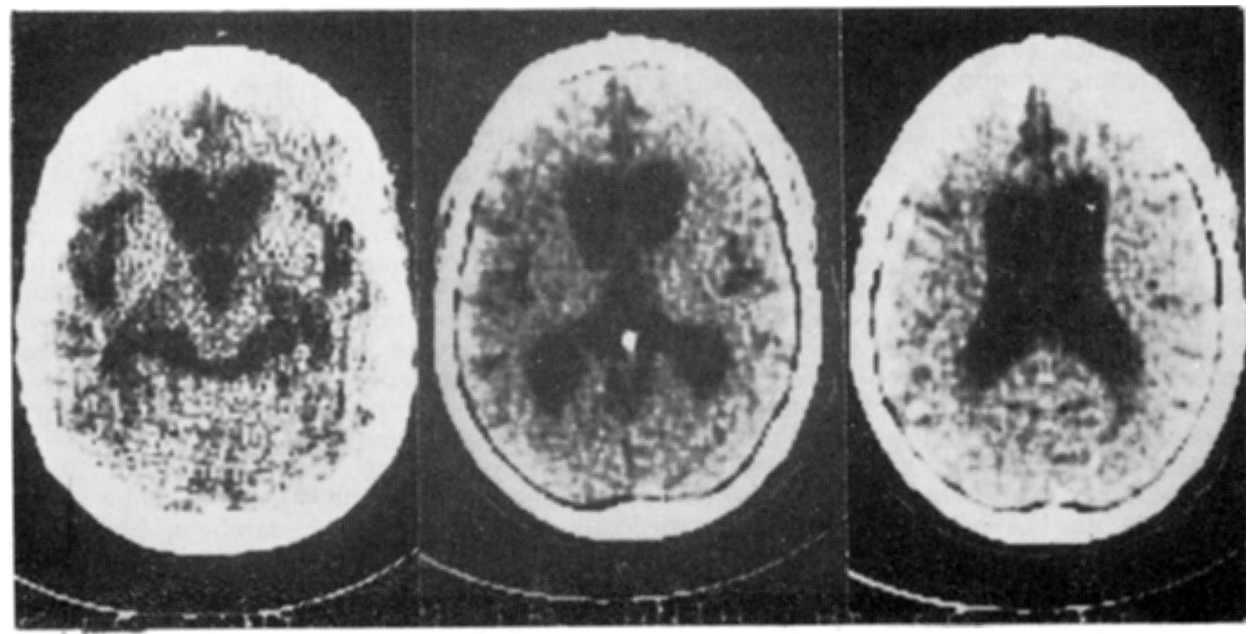

Fig. 2 - Caso MGO: exame tomográfico computadorizado do cranio.

material nāo mostrou alterações citológicas: 2 mononucleares $/ \mathrm{mm}: 3$ (65\% linfócitos, 30\% reticulomonócitos e 5\% macrófagos); bioquímica com $65 \mathrm{mg} \%$ de proténas totais (perfil albuminico), $75 \mathrm{mg} \%$ de glicose e $705 \mathrm{mg} \%$ de cloretos. A paciente permaneceu estavel por quase dois meses. Findo este periodo, veio a talecer em septicemia, depois de dispender cerca de 6 meses em estado vegetativo.

Obtido consentimento, o encéfalo foi removido. Pesava $870 \mathrm{~g}$, apresentando-se reduzido como um todo, sem alteraçōes do padrāo convolucional ou ná-formações vasculares. Os vasos do poligono de Willis encontravam-se permeáveis, com pequenas formaçōes ateromatosas endoteliais esparsas. Nos cortes coronais, não havia indícios de lesões parenquimatosas focais, antigas ou recentes. Os ventrículos laterais e o mediano estavam consideravelmente alargados. Os achados histopatológicos serão divulgados oportunamente, em publicação à parte. Placas neuríticas e degenerasão ncurofibrilar, contudo, estiveram ausentes.

\section{COMENTARIOS}

Considerações diagnósticas - Un dos aspectos mais marcantes do presente caso advém do contraste entre o traçado de vigilia normal, por um lado e, por outro, pelo adiantado estado demencial, refletido na intensa atrofia encefálica constatada radiologicamente. A paciente seguiu curso evolutivo idêntico ao de casos descritos como 'demência de tipo frontal' $76-78$, cujo substrato anatômico mais comum é a variante 'clássica' da doença de Pick 17,20,21,71,74,76-78,86,108,111. Observaçōes anátomo-clínicas têm sido quase unânimes ao demonstrar a distinção entre as doenças de Pick (DP) e Alzheimer (DA), em termos de seus respectivos padrōes EEGráficos. Na primeira, - EEG é, em geral, normal, pelo menos até os estágios finais. $\mathrm{Na}$ segunda, ao contrário, o ritmo alfa tende a desaparecer e dar lugar a ritmos-de-fundo lentificados e pouco reativos, dispostos nas faixas teta e delta, aos quais se sobrepõem alteraçōes episódicas focais, tais como surtos de ondas delta rítmicas, sincrônicas, de predominio frontal; ondas trifásicas e pontas e ondas agudas e bissincrônicas 16,21,23,32,46, $\mathbf{5 2 , 6 2 , 8 5 , 9 0 , 9 5 , 9 8 , 1 0 0}$. A quantidade de ondas delta nesses pacientes se correlaciona de maneira significativa com a reduçăo da densidade neuronal contada em territórios iso e allocorticais 16, por sua vez intimamente ligada aos principais índices de gravidade da condição demencial. A TC pôde igualmente fornecer informações adicionais relevantes, uma vez que na DP clássica afeta, principalmente, as fissuras sylvianas e inter-hemisférica, com relativa preservação dos sulcos corticais mais altos, configurando o padrão de 'atrofia dissociada' $36,71,108$. Nos estágios pré-terminais, a paciente exibiu combinação de sintomas de certo modo comum em determinados pacientes em processo demencial, na qual um incremento da atividade motora, particularmente da deambulação - que pode tornar-se incessante durante todo o periodo de vigília se acompanha de perda proporcional da fala e da linguagem, eventualmente culminando em sua completa extinção (mutismo). No caso, a acatisia se acompanhou de 
indiferença afetiva e apatia extremas, ao lado da tendência a pegar objetos próximos, aleatoriamente (hipermetamorfose), e levá-los à boca, aparentemente de modo compulsivo, configurando, assim, o quadro sindrômico de Klüver-Bucy 16,85.

Nos estágios finais, conservou-se a alternância dos ciclos sono-vigília, atestando a integridade, ao menos parcial, do prosencéfalo basal, do hipotálamo anterior e posterior, do subtálamo e do tegmento mesoprotuberancial. Além disso, as conexōes regionais do tronco encefálico - do bulbo à transição mesodiencefálica - se encontravam igualmente intactas, como demonstrado pelos reflexos óculo-cefálicos, céfalo-oculógiros, vestibulo-oculares, pupilares e de pestanejar 101. Por outro lado, a atitude em descorticaçāo bilateral, ainda que assimétrica, pareceu indicar acometimento supratentorial do tecido cortical rostralmente situado em relação ao sulco central. Finalmente, a integridade do reflexo de acompanhamento da mirada, aliada ao EEG normal, sugeriram ausência de alteraçóes significativas do córtex parieto-occipital, incluindo as projeções occipito-mesencefálicas 26. Alusōes a essa condição têm sido breves e escassas na literatura, não lhe dedicando os autores atenção à parte ('caso 3' de Muñoz-Garcia e Ludwin 76 e observações de Rosadini e Gentilomo 92). Como ven sendo enfatizado na DA30,55,72,77,99, o diagnóstico de certeza da DP é, também, histopatológico. Todavia, critérios de 'probabilidade' diagnóstica para a DP, semelhantes aos recentemente propostos para a $\mathrm{DA}^{30,55}$, seriam oportunos. Neste sentido, é possível que as demências de curso progressivo, senis ou pré-senis, marcadas por alterações da personalidade e acompanhadas de acatisia, reduçāo da produção lingüística, sindrome de Klüver-Bucy completa ou fragmentária e EEG normal ou pouco alterado em relação ao grau de demência, sejam expressăo maior da DP, especialmente em sua forma 'clássica' ou lobar. O achado tomográfico de padrāo dissociado de atrofia pode ser significativo para a confirmaçāo do diagnóstico, acentuando ainda mais o contraste hoje bem documentado entre a maior parte dos casos de DA e DP 20,22,77, $79,95,98$.

Caracterização sindrômica - Em 1972, Jennett e Plum 51, com base em revisão de literatura, reuniram na categoria 'estado vegetativo persistente' (EVP) um grupo de condiçōes clinicamente semelhantes, cujo denominador comum era dado por estado protraido de irresponsividade de olhos abertos, que se alternava a intervalos regulares com períodos de sono aparente, durante os quass os olhos se fechavam. Antes, diferentes autores cunharam nomes distintos para tais condições, relevando um aspecto ou outro que lhes parecesse mais ou menos significativo. A expressão oferecida por Jennett e Plum enfatizou os aspectos mais constantes da sindrome, ajudando a reduzir a confusão terminológica então dominante. Várias etiologias têm sido implicadas em sua produção 42,106 e o espectro de topografia das lesôes responsáveis é amplo, indo de afecções circunscritas dos lobos frontais 18 ou do tálamo 94 , por exemplo, até a destruição extensa do córtex cerebral e dos núcleos da base. Sob a designaçāo 'sindrome apallica', Kretschmer isolou a modalidade de EVP que se observa nos estágios terminais das demências de curso indolente e progressivo 57. Este parece ser o caso da paciente que relatamos. $\mathrm{O}$ aspecto mais notável dos EVPs é fornecido pelo contraste entre a aparência 'vigilante' dos olhos e a total irresponsividade comportamental. A rigor, esses pacientes não deveriam ser incluidos no diagnóstico do coma, que exige o fechamento dos olhos. Provavelmente, esse critério neuroftalmológico encerre importantes implicaçōes anatômicas e funcionais, não devendo ser reduzido a mero formalismo semântico. Assim, conforme demonstrado pelos pacientes em coma-alfa 'verdadeıro', isto é, por lesao do istmo do tronco cerebral, o aspecto do olhar e, mesmo, a própria vigília comportanental, parece estar na dependência de neurônios ali situados. Na realidade, essa fatia de tecido nervoso representa a extensāo caudal mínima da vasta região de orientação longitudinal que se prolonga rostralmente até o hipotálamo posterior e ao subtálamo 87. Destruída, advém o coma. Nos EVPs, ao contrário, esses neurônios istmicos persistem intactos em grande parte e, clinicamente, isso se manifesta pela possibilidade de abertura dos olhos. Investigaçöes post-mortem têm, de fato, confirmado tal inferência ao constatarem que, nesses casos, 0 tronco se acha preservado em relação aos hemisférios cerebrais 8,14,26,101. De modo geral, pacientes em EVP apresentam EEG isoelétrico ou dominado por ondas lentas de alta voltagem $3,9,10,40,82 \mathrm{e}$, tanto o fluxo sangüineo, como o metabolismo das estruturas supratentoriais, ficam reduzidos a menos de $25 \%$ do normal 46,47. Com base nesses dados, acreditamos que a inclusão de pacientes com lesōes pós-anóxicas ou traumáticas do córtex cerebral na categoria 'coma' ou 'coma-alfa' seja inapropriada. Sugerimos a denominação 'estado vegetativo-alfa' persistente (EVAP) para aqueles que, portando lesöes predominantemence supratentorais e, fugindo ao comum, exibanı traçados EEGráficos semelhantes ou indistingüiveis dos da vigilia normal. Essa associação pode ser tipicamente verificada nos estágios crônicos da lesão. $O$ termo 
'coma', entāo, continuaria reservado para abrigar aqueles pacientes irresponsivos de olhos fechados, não importando o padrão gráfico que venham a manifestar ao EEG. Assim definido, persistiria indicando comprometimento direto ou à distância dos neurônios do tegmento do istmo do tronco cerebral (Tabela 2).

\begin{tabular}{|c|c|c|c|}
\hline & Ausente & Presente & Correlatos anatômicos \\
\hline Coma-'!ento' & $\begin{array}{l}\text { vigilia comporta- } \\
\text { mental } \\
\text { ativação EEGrá- } \\
\text { fica } \\
\text { cognição }\end{array}$ & - - & lesão mesodiencefálica \\
\hline Coma-alfa & $\begin{array}{l}\text { vigilia comporta- } \\
\text { mental } \\
\text { cogniçăo }\end{array}$ & $\begin{array}{l}\text { EEG na faixa } \\
\text { affa, passivel de } \\
\text { ativaçảo eventual }\end{array}$ & $\begin{array}{l}\text { lesão tegmental } \\
\text { pontomesencefálica }\end{array}$ \\
\hline EVP convencional & $\begin{array}{l}\text { atjvaçāo EEGrá- } \\
\text { fica }\end{array}$ & EEG isoelêtrica & $\begin{array}{l}\text { lesão cortical extensa, dos } \\
\text { núcleos da base e do tála- } \\
\text { mo, com preservação rela- } \\
\text { tiva do tronco cerebral }\end{array}$ \\
\hline 'EVAP' & cognição & $\begin{array}{l}\text { vigrilia comporta- } \\
\text { mental } \\
\text { EEG na faixa } \\
\text { alfa, passivel de } \\
\text { ativaçăo eventual }\end{array}$ & $\begin{array}{l}\text { lesão predominante das ca- } \\
\text { madas infragranulares do } \\
\text { córtex, com preservação re- } \\
\text { lativa de núcleos da base, } \\
\text { tálamo e tronco cerebral }\end{array}$ \\
\hline
\end{tabular}

Tabela 2 - Caracteristicas clinicas $e$ fisiopatologicas dos estados de coma e vegetativo persistente. EVP, estado vegetativo persistente. EVAP, estado vegetativo-alfa persistente. Vigilia comportamental: abertura dos olhos, espontanea on evocada, com duracão variável, podendo-se acompanhar de movimentos de pestanejar e centragem dos olhos. Ativacão EEGráfica, atividade rápida de baixa voltagem que habitualmente coexiste com a vigilia comportamental, de duraçāo variável e iguxlmente evocavel por estimulacão sensitivo-sensorial.

A variabilidade espontânea e a reatividade do traçado da paciente relatada aproxima-a dos casos de coma-alfa por lesão istmica. Todavia, o quadro anátomo-clínico é idêntico ao observado nos EVPs pós-anóxicos ou traumáticos, onde o EEG costuma ser isoelétrico. Trata-se, portanto, de associação anátomo-eletroclinica peculiar. Esperemos que novos casos sejam reportados, para que seja possivel uma compreensão mais exata do significado clínico, bem como dos mecanismos fisiopatológicos envolvidos na sua produção.

Ayrudecimentos - Estamos em débito para com os Drs.: Hamilton Clemente, que gentilmente realizou o exame do LCR; Rogerio Paysano Marrocos (Hospital Dr. P. Pinel), pelo auxílio na conđução do caso; Sergio Luis Schmitt (Instituto de Biofisica, UFRJ), pelas criticas e sugestōes ao manuscrito; Felicio Jahara (Santa Cașa de Misericórdia, RJ), pelo exame tomográfico. Sem eles, este trabalho nāo teria sido realizado.

\section{REFERENCIAS}

1. Austin EJ, Bhagat A, Dodrill CD, Wilkus RJ - EEG rhythms of alpha frequency in a patient with hyperosmolar diabetic coma followed by partial recovery. Electroenceph Clin Neurophysiol 57:61P, 1984.

2. Austin EJ, Wilkus RJ, Longstreth W'T - Etiology and prognosis of alpha-coma. Neurology $38: 773,1988$.

3. Autret A. Carpier H, Tommasi M, Jouvet M, Schott B - Etude physiopathologique et neuropathologique d'un syndrome de décortication cérébrale. Rev Neurol 131:491, 1975.

4. Batini C, Moruzzi G, Palestini M, Rıssi GF, Zanchetti A - Persistent patterns of wakefulness in the pretrigeminal midpontine preparation, Science 128:30, 1958.

5. Boonstra $S$, Notermans SLH - The EEG in 17 cases of lesions of the brain-stem. established post-mortem. Electroenceph Clin Neurophysiol 23:496, 1967. 
6. Brain $\mathrm{R} \rightarrow$ The physiological basis of consciousness: a critical review, Brain 81:426, 1958 .

7. Bricolo A, Gentilomo A, Rosadini G, Rossi GF - Long-lasting post-traumatic unconsciousness. Acta Neurol Scand 44:512, 1968.

8. Brierley JB, Adams JH, Graham DI, Simpson JA - Neocortical deaih after cardiac arrest: a clinical, neurophysiological, and neuropathological report of two cases. Lancet $2: 560,1971$.

9. Cairns H, Oldfield RC, Pennybacker JB, Whitteridge $D$ - Akinetic nutism with an epidermoid cyst of the 3rd. ventricle. Brain 64:273, 1941.

10. Carrol WM, Mastaglia FL - «Locked-in coma» in postinfective polyneuropathy. Arch Neurol $36: 46,1979$.

11. Carrol WM, Mastaglia FL - Alpha and beta coma in drug intoxicatiou uncomplicated by cerebral hypoxia. Electroenceph Clin Neurophysiol 46:95, 1979.

12. Chase TN, Moretti L, Prensky AL - Clinical and electroencephalographic manifestations of vascular lesions of the pons. Neurology 18:357, 1968.

13. Chatrian GE, White LE, Shaw CM - EEG pattern resembling wakefulness in unresponsive decerebrate state following traumatic brain-stem infarct. Electroenceph Clin Neurophysiol $16: 285,1964$.

14. Chokroverty $\mathrm{S}$ - "Alpha-like» rhythms in electroencephalograms in coma after cardiac arrest. Neurology 25:655, 1975.

15. Collins AT, Chatrian GE - EEG rhythm of alpha frequency in a 22-month-old child. Neurology 30:1316, 1980.

16. Constantinidis $\mathrm{J}$, Krassoievitch $\mathrm{M}$, Tissot $\mathrm{M}$ - Corrélations entre les perturbations électroencéphalographiques et les lésions anatomo-histologiques dans les démences. Encéphale 58:19, 1969.

17. Cummings JL, Duchen LW - Kluvers-Bucy syndrome in Pick disease: clinical and pathologic correlations. Neurology 31:1415, 1981.

18. Damasio AR, van Hoesen GW - Emotional disturbances associated with focal lesions of the limbic frontal lobe. In Heilman KM, Satz P (eds): Neuropsychology of Human Emotion. Guilford, New York, 1983, pg 85.

19. Dehaene I - Bilateral thalamo-subthalamic infarction. Acta Neurol Belg 82:253, 1981.

20. Delay J, Brion S, Escourolle R - L'opposition anatomo-clinique des maladies de Pick et d'Alzheimer: étude de 38 cas. Presse Méd 65:1495, 1957.

21. Delay $\mathrm{J}$, Brion $\mathrm{S}$, Escourolle $\mathrm{R}$ - L'opposition anatomo-clinique des maladies de Pick et d'Alzheimer: valeur diagnostique des examens complementaires. Presse Méd 65:1515, 1957.

22. Delay $J$, Brion $S$, Escourolle $R$ Les lésions des noyaus gris et du tronc cérébral dans la maladie de Pick. Encéphale 49:463, 1960.

23. Delay J, Neveu P, Lerique $P$ - Sur quelques résultats concordants de la pneumo-encéphalographie et de l'électru-encéphalographie dans le diagnostic des atrophies cérébrales. Rev Neurol $76: 263,1944$.

24. Deleu $\mathrm{D}$, de Keyser $\mathrm{J}$ - Flunitrazepam intoxication simulating a structural brainstem lesion. J Neurol Neurosurg Psychiat 50:236, 1987.

25. Dick JPR, Gledhill RF, Sheehy MP - Loss of consciousness in migraine: clinical and EEG findings during an attack. Ann Neurol 19:514, 1986.

26. Dougherty $\mathrm{JH}$, Rawlison DG, Levy DE, Plum F - Hypoxic-ischemic brain injury and the vegetative state: clinical and neuropathologic correlation. Neurology 31:991, 1981.

27. Façon E, Steriade M, Wertheim N- Hypersomnie prolongée engendrée par des lésions bilatérales du système activateur médial: le syndrome thrombotique de la bifurcation du tronc basilaire. Rev Neurol 98:117, 1958.

28. Ferguson JM, Bennett DR: Sleep in a patient with a pontine infarction. Electroenceph Clin Neurophysiol $36: 210,1974$.

29. Flügel KA - Alphakoma, Pseudoalpha-Koma und Alpha-Pseudokoma: Zur Differentialdiagnose des sogenannten Alphakomas. Fortschr Neurol Psychiat 50:371, 1982.

30. Fox JH, Penn R, Clasen $R$ - Pathological diagnosis in clinically typical Alzheimer's disease. N Eng $J$ Med $313: 1419,1985$.

31. Fung PC, Tucker RP - Alpha rhythm and alpha-like activity in coma. Clin Electroenceph 15:167, 1984.

32. Gordon EB, Sim M - The EEG in presenile dementia. J Neurol Neurosurg Psychiat $30: 285,1967$.

33. Grigg MM, Kelly MA, Celesia GG, Ghobrial MW, Ross ER - Electroencephalographic activity after brain death. Arch Neurol 44:948, 1987.

34. Grindal AB, Suter C - «Alpha-pattern coma» in high voltage electrical injury. Electroenceph Clin Neurophysiol 38:521, 1975. 
35. Grindal AB, Suter C, Martine\% AJ - Alpha pattern coma: 24 cases with 9 survivors. Ann Neurol 1:371, 1977.

36. Groen JJ, Endtz LJ - Hereditary Pick's disease: second examination of a large family and discussion of other hereditary cases with particular reference to electroencephajography and computerized tomography. Brain 105:443, 1982.

37. Guiloff RJ, Cobb WA - Diagnosis of coma. Lancet 2:1351, 1977.

38. Gurvitch AM, Zarzhetsky Yu V, Trush VD, Zonov VM - Experimental data on the nature of postressucitat.on alpha frequency activity. Electroenceph Clin Neurophys.el $58: 426,1984$.

39. Guterman B, Sebastian P, Sodha N - Recovery from alpha-coma after lorazepam overdose. Clin Electroenceph 12:205, 1981.

40. Hansotia PL - Persistent vegetative state: review and report of electrodiagnostic studies in eight cases. Arch Neural 42:1048, 1985.

41. Hari R, Sulkava R, Haltia $M$ - Brainstem auditory evoked responses and alpha-pattern coma. Ann Neurol 11:187, 1982.

42. Higashi $K$, Hatano M, Abiko S. Ihara $K$, Katayama S, Wakuta $Y$, Okamura T, Yamashita $\mathbf{T}$ - Five-year follow-up study of patients with perssstent vegetative state. J Neurol Neurosurg Psychiat 44:552, 1981.

43. Homan RW, Jones MG - Alpha-pattern coma in a 2-month-old child. Ann Neurol 9:611, 1981.

44. Hughes JR, Cayaffa J, Leestma J, Mizuna $Y$ - Alternating «waking» and «sleep» EEG patterns in a deep comatose patient. Clin Electroenceph 3:86, 1972.

45. Ingvar $\mathrm{DH}$ - Cerebral blood flow and metakolism in complete apalic syndromes, in states of severe dementia and in akinetic muc.sm. Acta iveurol Scand 49:233, 1973.

46. Ingvar DH, Brun A - Das komplette apalıscne syndrom. Arch Psychiat Nervenkr 215:219, 1972 .

47. Ingvar DH, Brun A, Hagberg B, Gustafson L - Reg.onal blood flow in the dominant hemisphere in conturmed cases of Alzheimer's wsease, Psck's disease, and muiti-infast dementia: relationship to clinical symptomatology and neuropathological findings, in Katzman K, Terry RD, Bick KL (eds): Aizıeımer's Disease: Senıle Dementia and Related Disorders, Raven, New York, 1978, pg 203.

48. Iragui VJ, McCutchen CB - Physiologic and prognostic significance of «alpha-cona». $J$ Neurol Neurosurg Psychiat 46:632, 1983.

49. Jasper HH, Andrews HL - Electroencephalography: III. Normal differentiation of occipital and precentral regions in man. Arch veurol Psychiat 39:96, 1938.

50. Jasper HH, van Buren HL - Interrelationship between cortex and subcortical structures: clinical and electroencephalographic studies. Electroenceph Clin Neurophysiol suppi $4: 168,1953$.

51. Jennett B, Plum F - Persistent vegetative state: a syndrome in search of a name. Lancet 1:734, 1972.

52. Jóhannesson G, Brun A, Gustafson I, Ingvar DH - EEG in presenile denientia related to cerebral blood flow and autopsy findings. Acta Neurol Scand 56:89, 1977.

53. Jouvet M. Michel F, Courjon J - Sur un stade d'activité électrique cérébrale rapide au cours du sommeil phys.ologique. C R Soc Biol (Paris) 153:1024, 1959.

54. Kaada BR, Karkmark W, Stokke O - Deep coma associated with desynchronization in EEG. Fitctroencenh Clin Neurophysiol 13:785, 1961.

55. Katzman $k$ - Alzheimer's disease. N Eng $J$ Med 314:964, 1986.

56. Keane JR, Zaias B, Itabashi HH - Levator-sparing oculomotor nerve palsy caused by a solitary midbrain metastasis. Arch Neurol 41:210, 1984.

57. Kretschmer E - Das apallische Syndrom. Z ges Neurol Psychiat 169:576, 1940.

58. Kuroiwa $Y$, Furukawa $T$ - EEG prognostication in drug-related alpha-coma. Arch Neurol 38:200, 1981.

59. Kuroiwa $Y$, Furukawa T, Inaki $K$ - Recovery from drug-induced alpha-coma. Neurology $31: 1359,1981$.

60. Lee SI - Coexistence of triphasic waves and alpha-coma pattern. Electroenceph Clin Neurophysiol $51: 108,1981$.

61. Lersch DR, Kaplan AM - Alpha-pattern coma in childhood and adolescence. Arch Neurol 41:68, 1984.

62. Letemendia F, Pampiglione $\mathbf{G}$ - Clinical and electroencephalographic observations in Alzheimer's disease. J Neurol Neurosurg Psychiat 21:167, 1958.

63. Lindsley DB, Bowden JW, Magoun HW - Effects upon the EEG of acute injury to the brain stem activating system. Electroenceph Ctin Neurophysiol 1:475, 1949.

64. Loeb C - Electroencephalographic changes during the state of coma. Electroenceph Clin Neurophysio] 10:589, 1958. 
65. Loeb C, Poggio GF - Electroencephalogram in a case with ponto-mesencephalic hemorrhage. Electroenceph Clin Neurophysiol 5:295, 1953.

66. Loeb C, Rosadini G, Poggio GF - Electroencephalograms during coma: normal and borderline records in 5 patients. Neurology 9:610-18 (1959).

67. Lundervold A - EEG in patients in coma due to localized brain lesions. Handbook Electroenceph Clin Neurophysiol 12:37, 1975.

68. Lundervold A, Hauge $T$, Loken AC - Unusual EEG in unconscious patient with brain stem atrophy. Electroenceph Clin Neurophys ol 8:665, 1956.

69. Marshall DW - Recovery from drug-induced coma. Neurology 34:406, 1984.

70. Masson C, Mear JY, Masson M, Cambier J - «Ictus hypersomnique» symptomatique d'une hémorragie thalamique postérieure. Presse Méd 16:79, 1987.

71. MeGeachie R, Fleming J, Sharer L, Hyman RA - Diagnosis of Pick's disease by computed tomography. J Comput Assist Tomogr 3:113, 1979.

72. McKahn G, Drachman D, Folstein M, Katzman R, Price D, Stadlan EM - Ciinical diagnosis of Alzheimer's disease: report of the NINCDS-ADRDA Work Group under the auspices of the Department of Health and Human Services Task Force on Alzheimer's disease. Neurology $34: 939,1984$.

73. Meienberg $O$, Mumenthaier $M$, Karbowski $K-$ Quadriparesis and nuclear cculomotor palsy with total bilateral ptosis mimicking coma: a mesencephalic «locked-in syndrome»"' Arch Neurol 36:708, 1979.

74. Mingazzini $\mathbf{G}$ - On aphas:a due to atrophy of the cerebral convolutions. Brain $36^{\circ}: 493$, 1914.

75. Moruzzi G, Magoun HW - Brain stem reticular formation and activation of the EEG. Electroenceph Clin Neurophysiol 1:455, 1949.

76. Muñoz-Garcia D, Ludwin SK - Classic and reneralized variants of Pick's disease: a clinicopathological, ultrastructural, and immunocytochemical comparative study. Ann Neurol 16:467, 1984 .

77. Neary D, Snowden JS, Bowden DM, Sims NR, Mann DMA, Benton JS, Northen B, Yates PO, Davison AN - Neuropsychological syndromes in presenile dementia due to cerebral atrophy. J Neurol Neurosurg Psychiat 49:163, 1986.

78. Neary D, Snowden JS, Northen B, Goulding $P$ - Dementia of frontal lobe type. J Neurol Neurosurg Psychiat 51:353, 1988.

79. Neumann MA - Pick's disease. J Neuropath Exp Neurol 8:255, 1949.

80. Neumann MA, Cohn $R$ - Reticular formation and coma in man. Trans Am Neurol Ass $89: 235,1964$.

81. Neundörfer B, Meyer-Wahl L, Meyer JG: Alpha-EEG und Bewustlosigkeit: ein kasuistischer Beitrag zur lokaldiagnostischen Bedeutung des $\alpha$-EEG beim bewustlosen Patienten. Z EEG-EMG 5:106, 1974.

82. Nyström S - A case of decortication following a severe head injury. Acta Neurol Scand $35: 101,1960$.

83. Obeso JA, Iragui MI, Marti-Masso JF, Maravi E, Teijeira JM, Carrera N, Teijeira $\mathbf{N}$ - Neurophysiological assessment of alpha pattern coma. J Neurol Neurosurg Psychiat $43: 63,1980$.

84. Obrador S, Reinoso-Suarez F, Carbonell J, Cordoba A, Martinez-Moreno E, Navarro V, Oliva H, Oliveros JC - Comatose state maintained during eight years following a vascular ponto-mesencephalic lesion. Electroenceph Clin Neurophysiol 38:21, 1975.

85. Obrist WD - Electroencephalography in aging and dementia. In Katzman R, Terry RD, Bick KKL (eds): Alzheimer's Disease: Senile Dementia and Related Disorders. Raven, New York, 1978, pg 227.

86. Pilleri G - The Kluver-Bucy syndrome in man: a clinico-anatomical contribution to the function of the medial temporal lobe structures. Psychiat Neurol (Basel) 152:65, 1966.

87. Plum F, Fosner J - The Diagnosis of Stupor and Coma. Ed 3. Dairs, Philadelphia, 1980.

88. Pulst SM, Lombroso CT - External ophtalmoplegia, alpha and spindle coma in imipramine overdose: case report and review of the literature. Ann Neurol 14:587, 1983.

89. Radermecker J - Severe acute necrosis of the pons with long survival: electro-clinical symptoms and absence of cerebral lesions. Electroenceph Clin Neurophysiol 23:281, 1967.

90. Rae-Grant A, Blume W, Lal C, Hachinski VC, Fisman M, Merskey H - The electroencephalogram in Alzheimer-type dementia. Arch Neurol 44:50, 1987.

91. Roger J, Roger A, Gastaut H - Electro-clinical correlations in 30 cases of vascular syndromes of the brain and brain stem. Electroenceph Clin Neurophysiol 16:164, 1954.

92. Rosadini G, Gentilomo A - Sleep EEG patterns during the apallic syndrome. Electroenceph Clin Neurophysiol 25:87, 1968. 
93. Routtemberg A - The two-arousal hypothesis: reticular formation and limbic system. Psychol Rev 75:51, 1954.

94. Segarra $J$ - Cerebral vascular disease and behavior: $I$. The syndrome of the mesencephalic artery (basilar artery bifurcation). Arch Neurol 22:408, 1970.

95. Sheridan PH, Sato S, Foster N, Bruno G, Cox C, Fedio P, Chase TN - Relation of EEG alpha background to parietal lobe function in Alzheimer's disease as measured by positron emission tomography and psychometry. Neurology 38:747, 1988.

96. Sieben G, de Reuck J, Vander Eeecken H - Thrombosis of the mesencephalic artery: a clinico-pathologic study of two cases and its correlation with the arterial vascularization. Acta Neurol Belg 77:151, 1977.

97. Silverman D - Retrospective study of EEG in coma. Electroenceph Clin Neurophysiol $15: 486,1963$.

98. Stigsby B, Jóhannesson G, Ingvar DH - Regional EEG analysis and reg.onal cerebral blood flow in Alzheimer's and Pick's disease. In Katzman R, 'Terry RD, Bick KL (eds): Alzheimer's Disease: Senile Dementia and Related Disorders. Raven, New York, 1978.

99. Sulkava R, Haltia M, Pateau A, Wikström J, Palo J - Accuracy of clinical diagnosis in primary degenerative dementia: correlation with ncuropathological findings. J Neurol Neurosurg Psychiat 46:9, 1983.

100. Swain JM - Electroencephalographic abnormalities in presenile atrophy. Neurology $9: 722,1959$.

101. Tavy DLJ, van Woerkom TCAM, Buts GTAM, Endtz LJ - Persistence of the blink reflex to sudden illumination in a comatose patient. Arch Neurol 41:323, 1984.

102. Thompson GN, Nielsen JM - Area essential to consciousness: cerebral localization of conscluusness as established by neuropathological studies. J Am Med Ass 137:285, 1948.

103. Tomassen W, Kamphuisen HAC: Aipha coma. $s$ Neurol Sci 76:1, 1986.

104. Turazzi S, Bricolo A - Acute pontine syndromes following head injury. Lancet 2:62, 1977.

105. Vignaendra V, Wilkus RJ, Copass MK, Chatrian GE - Electroencephalographic rhythms of alpha frequency in comatose patients after cardiopulmonary arrest. Neurology 24:582, 1974 .

106. Walshe TM, Leonard C - Persistent vegetative state: extension of the syndrome to include chronic disorders. Arch Neurol 42:1045, 1985.

107. Walter WG, Griffiths GM, Nevin S - The electro-encephalogram in a case of pathological sleep due to hypothalamic tumor. Br Med J 1:107, 1939.

108. Wechsler AF, Verity A, Rosenschein S - Pick's disease: a clinical, computed tomograph'c, and histologic study with Golgi impregnation observations. Arch Neurol 39:287, 1982.

109. Westmoreland B, Klass D, Sharbrough FW, Reagan TJ - Alpha coma: electroencephalographic, clinical, pathological and etiologic correlations. Arch Neurol 32:713, 1975.

110. Wilkus RJ, Harvey F, Ojeman LM, Lettich E - Electroencephalogram and sensory evoked potentials: findings in an unresponsive patient with pontine infarct. Arch Neurol 24:538, 1971 .

111. Wisniewski HM, Coblents JM, Terry RD - Pick's disease: a clinical and ultrastructural study. Arch Neurol 26:97, 1972 